\title{
ANÁLISE DE SOBREVIVÊNCIA DE EMPRESAS: UM ESTUDO LONGITUDINAL DA COORTE DE 2007 NO RIO GRANDE DO SUL
}

Carlos Hernán Rodas Céspedes ${ }^{1}$ Adelar Fochezatto ${ }^{2}$ Leandro Justino Pereira Veloso ${ }^{3}$

Resumo: Este trabalho propõe uma pesquisa empírica sobre a sobrevivência das empresas do Rio Grande do Sul para o período 2007-2013. É aplicado o procedimento de Kaplan-Meier para obter as funções de sobrevivência das empresas, conforme o local, a atividade econômica e o tamanho. Da mesma forma, aplica-se o procedimento de Cox, com a finalidade de determinar o efeito do tamanho das empresas sobre o tempo de sobrevivência. Os resultados obtidos mostram que a sobrevivência é relativamente maior: nas empresas com maior número de pessoas ocupadas; no setor da indústria de transformação e atividades financeiras; e localizadas na região Nordeste do Rio Grande do Sul.

Palavras-chave: Economia regional. Demografia de empresas. Sobrevivência. Kaplan-Meier. Modelo de Cox.

\section{SURVIVAL ANALYSIS OF BUSINESS: A LONGITUDINAL STUDY FOR RIO GRANDE DO SUL STATE, 2007}

Abstract: This paper proposes an empirical research on the survival of the companies of Rio Grande do Sul State for the period 2007-2013. The Kaplan-Meier procedure is applied to obtain the functions of survival of the companies, according to the geographic location, the economic activity and the size. Then, the Cox procedure is applied, in order to determine the effect of the size of the companies on the survival time. The results obtained show that survival is relatively higher: in the companies with the largest number of employed persons; in the manufacturing industry and financial activities; and located in the Northeast region of Rio Grande do Sul state.

Keywords: Regional economics. Business demography. Survival. Kaplan-Meier. Cox model.

\section{ANALISIS DE SUPERVIVIENCIA DE EMPRESAS: UN ESTUDIO LONGITUDINAL PARA EL ESTADO DE RIO GRANDE DO SUL, 2007}

Resumen: Este trabajo es una propuesta de investigación empírica sobre la supervivencia de las empresas de Rio Grande do Sul correspondiente al periodo entre 2007 e 2013. Se aplica el procedimiento metodológico de Kaplan-Meier con la finalidad de establecer las funciones de supervivencia de las empresas según su tamaño, localización y actividad económica. Igualmente, es aplicado el procedimiento metodológico de Cox con el objetivo de determinar el efecto del

\footnotetext{
1Universidade Federal do Pampa, Departamento de Ciências Econômicas, Santana do Livramento/RS, Brasil, carloscespedes@unipampa.edu.br, http://orcid.org/0000-0002-6347-4970

${ }^{2}$ Pontifícia Universidade Católica do Rio Grande do Sul, Escola de Negócios, Porto Alegre/RS, Brasil, adelar@pucrs.br, http://orcid.org/0000-0001-7192-3986

${ }^{3}$ Universidade Federal do Rio de Janeiro, Departamento de Ciência da Computação, Rio de Janeiro/RJ, Brasil, leandrojpveloso@gmail.com, https://orcid.org/0000-0002-9153-6737
} 
tamaño de las empresas sobre la supervivencia. Los resultados conseguidos demuestran que la supervivencia es relativamente mayor en las empresas con el mayor número de personas ocupadas, en las del sector de la industria de transformación y del sector financiero, y en las que se encuentran localizadas en la región Nordeste de Rio Grande do Sul.

Palabras clave: Economía regional. Demografía de empresas. Supervivencia. Kaplan-Meier. Modelo de Cox.

\section{Introdução}

Quando o empreendedor ou o gestor de recursos decide abrir uma empresa, seu comportamento é guiado pela expectativa de lucros e/ou pelo desejo de ampliar sua participação no mercado. Se tal expectativa e desejo não são correspondidos e eventuais perdas ocorrem e persistem, o encerramento do negócio surge como a alternativa mais provável.

No Brasil, segundo o SEBRAE (2016, p.16) a taxa de sobrevivência das empresas com até dois anos de atividade tem evoluído favoravelmente passando de $75,6 \%$ em 2007, para 76,2\% em 2010, e alcançando 76,6\% em 2012, sinalizando o clima de estabilidade para os negócios nos dois últimos dois anos. Em termos comparativos, esses registros se aproximam da taxa de sobrevivência das empresas de alguns países desenvolvidos, embora a comparação padeça de diferenças metodológicas e desajustes temporais ${ }^{4}$. Por exemplo, no ano de 2007, para o prazo de dois anos em atividade, as empresas europeias e americanas apresentaram os seguintes registros: Áustria, 71,8\%; Canadá, 76,4\%; Estados Unidos, 75,5\%; Espanha, 68,7\%; Finlândia, 64,4\%; Itália, 68,2\%; e Portugal, 52,2\%.

Embora a menor taxa de sobrevivência guarde relação com uma maior taxa de rotatividade das empresas e esta, por sua vez, influencie a competitividade dos empreendimentos, na medida em que as empresas menos eficientes são substituídas por empresas mais eficientes, uma taxa de sobrevivência mais elevada afeta positivamente o crescimento de longo prazo da empresa, desde que a maior permanência no mercado aumente as chances de a empresa vir a desenvolver uma estratégia em favor da qualificação da mão de obra e da inovação.

Com tal perspectiva, este ensaio analisa a taxa de sobrevivência de empresas no Rio Grande do Sul. O objetivo é mensurar a sobrevivência de empresas no período entre 2007 e 2013. Para tanto, utiliza-se a técnica não paramétrica de Kaplan-Meier, de modo a efetuar um acompanhamento da vida das empresas em

\footnotetext{
${ }^{4}$ Segundo o SEBRAE, a metodologia da OECD utilizada para o cálculo da taxa de sobrevivência inclui somente as empresas empregadoras, enquanto que os estudos do SEBRAE incluem também as empresas sem nenhum empregado.
} 
intervalos anuais até o fechamento acontecer. Este acompanhamento é feito segundo o tamanho do estabelecimento, a sua localização geográfica e segundo a atividade produtiva. Também, será utilizada a técnica semiparamétrica de Cox, com a finalidade de testar o efeito do tamanho dos estabelecimentos sobre o risco de fechamento das empresas.

Os dados utilizados neste estudo foram obtidos junto à Relação Anual de Informações Sociais do Ministério de Trabalho e correspondem a informações identificadas e únicas que permitem o acompanhamento das empresas produtivas do Rio Grande do Sul ao longo do período aludido anteriormente.

$\mathrm{Na}$ próxima seção, é apresentada a revisão da literatura sobre a sobrevivência de empresas; na terceira seção são apresentados os procedimentos metodológicos adotados; na quarta seção são mostrados os resultados do estudo; e, finalmente, na última seção são apresentadas as conclusões.

\section{Revisão da literatura}

De maneira geral, a sobrevivência de uma empresa recém-entrante na indústria é afetada negativamente pela intensidade do capital e pelas economias de escala ali vigentes. Porém, em determinadas condições, podem ocorrer situações divergentes dessa. Audretsch (1991), ao avaliar o impacto do regime tecnológico 5 sobre a longevidade da empresa recém-entrante na indústria, constata que é possível as firmas pequenas ingressarem e se posicionarem em indústrias intensivas em capital e com economias de escala. Essa situação depende do regime tecnológico vigente e da diferença entre os prazos de sobrevivência avaliados.

Como apontado por Weiss (1976 e 1979, apud Audretsch,1991), empresas com capacidade subótima (menor que a escala eficiente mínima) podem ser promovidas em indústrias cujo preço é maior que o custo médio mínimo; assim, quanto maior é essa diferença, maior é a probabilidade de firmas recentemente ingressadas sobreviverem. "The probability of any given firm, [...] remaining in industry [...] is essentially determined by the extent to which a firm is burdened with an inherent size disadvantage, the degree to which the industry price is elevated beyond average cost at the minimum efficient scale (MES) level of output, and the probability of innovative activity" (AUDRETSCH,1991, p.444).

\footnotetext{
${ }^{5}$ Marsili (2002) entende por regime tecnológico o ambiente tecnológico no qual uma empresa opera, mais especificamente, define as propriedades do processo de aprendizagem, as fontes do conhecimento e a natureza do conhecimento associado com o processo de inovação das firmas. São distinguidos dois tipos de regime tecnológico: um regime empreendedor que facilita a inovação da empresa que nasce e um regime rotineiro que facilita a inovação das incumbentes em uma indústria.
} 
O autor supracitado considera que a possibilidade de a empresa recémentrante atingir o nível de produto correspondente à escala eficiente mínima fica concretizado em caso do regime tecnológico em vigência favorecer o ingresso de pequenas empresas inovadoras e desfavorecer a atividade inovadora das incumbentes. "An entrepreneurial regime is one that is favorable to innovative entry and unfavorable to innovative activity by established firms; a routinized regime is one in which the conditions are the other way around" (AUDRETSCH, 1991, p.444).

Por outro lado, Jovanovic (1982) destaca o processo de aprendizagem a partir da própria experiência e dos níveis de eficiência alcançados desde ingresso da empresa no mercado. Segundo o autor, depois de completada a fase da aprendizagem, a taxa de sobrevivência aumenta conforme a idade e o tamanho da empresa.

Na mesma linha, o trabalho de Fotopoulos e Louri (2000) invoca as ideias de Jovanovic (1982) quando, na avaliação da sobrevivência empresarial do setor manufatureiro grego por meio de um modelo de risco/sobrevivência e dando destaque ao papel jogado pelo espaço geográfico (Grande Atenas versus o resto), salienta o processo de aprendizagem da empresa. Além de distinguir o papel outorgado às áreas metropolitano-urbanas enquanto incubadoras de empresas ou espaços geográficos adequados para inovar e interagir com outras firmas, os autores utilizam o modelo de Cox para explicar a sobrevivência a partir de um conjunto de variáveis, como, por exemplo: tamanho dos estabelecimentos, crescimento da empresa, lucratividade e alavancagem.

Ainda sobre o efeito espacial, o trabalho de Wennberg e Lindqvist (2010) investiga o efeito que aglomerações regionais de unidades produtivas industriais produziram sobre o desempenho e a sobrevivência de empresas. A partir de dados da economia sueca para o período de 1993-2002, evidenciaram que os empreendimentos localizados próximos dessas aglomerações ${ }^{6}$ eram capazes de criar mais empregos, impostos e maiores salários, não descartando o incremento na rivalidade produtiva e competitiva gerada pelos novos entrantes.

Num estudo mais recente sobre os determinantes da sobrevivência empresarial, Martinez (2016) também abordou a questão da aprendizagem levantada por Fotopoulos e Louri (2000), referindo-se de forma específica ao modelo de mobilidade empresarial com aprendizagem passiva de Jovanovic (1982).

\footnotetext{
${ }^{6}$ Marshall (1920 apud Wennberg e Lindqvist, 2010) sugeriu benefícios junto das aglomerações através dos ganhos de eficiência relacionados com a especialização industrial, agrupamento de mão de obra especializada, e transbordamento do conhecimento.
} 
Tsvetkova et al (2014) estudaram, entre outros, a influência das regiões inovadoras sobre o risco de fechamento das empresas por meio de um modelo de regressão logística de sobrevivência. Os resultados sugeriram uma relação de causalidade negativa entre a inovação e a sobrevivência dos estabelecimentos de menor tamanho, de um a três trabalhadores. Contudo, verificaram que à medida que os estabelecimentos cresceram esse efeito negativo diminuiu.

Por outro lado, Callejon e Ortún (2009) procuraram diferenciar dois tipos de empreendedores: por um lado, os que estão comprometidos com o crescimento e, por outro lado, os que não realizam um aporte significativo para essa finalidade e, como tal, comprometem a sua sobrevivência. Nesse estudo referem-se a um resultado não conclusivo, e até surpreendente, do Global Entrepreneurship Monitor a respeito das microempresas individuais, sugerindo que os empreendimentos individuais por necessidade representam um indicador negativo da economia. Já os empreendimentos de porte maior, procuram se expandir através de investimentos em capital físico e humano, como é desejável, e com isto demonstram maior ambição de crescimento e tendem a apresentar um maior horizonte de vida. (CALLEJON e ORTUN, 2009, p.172).

Ao salientar que a sobrevivência da empresa está relacionada com uma gestão eficiente e com capacidade de oferecer emprego de qualidade, os autores supracitados referiram-se ao trabalho de Salas y Sanchez-Asín (2009 apud Callejon e Ortún, 2009, p.174), no qual se destaca a possibilidade sugerida pelo estudo do Global Entrepreneurship Monitor, isto é, os empreendimentos com pessoal assalariado estão associados com maiores níveis de produtividade e trabalhadores mais capacitados, enquanto que os empreendimentos individuais estão associados a menores níveis de produtividade. (CALLEJON e ORTUN, 2009, p.174).

Indo ao encontro de modelos e resultados de procedimentos aplicados para estudar a sobrevivência de empresas, o estudo de Martinez (2006) realizado junto às empresas industriais de Cali, na Colômbia, no período 1994-2003, propõe, entre os determinantes da sobrevivência: o ciclo de vida, o nível de concentração do mercado e o regime tecnológico das indústrias. Por estrato de tamanhos, a representação de Kaplan-Meier evidenciou que as microempresas apresentaram a menor expectativa de sobrevivência, seguidas pelas pequenas empresas e, depois, pelas empresas grandes.

Martinez (2006) também encontrou que as empresas que ingressaram em mercados mais concentrados apresentaram níveis maiores de sobrevivência que as 
que ingressaram em estruturas menos concentradas. No entanto, com base no modelo de duopólio Stackelberg, o resultado encontrado permitiu ao autor argumentar que a empresa líder liberava o ingresso de empresas menores para capturar a demanda residual do mercado, portanto, a concentração de mercado podia não atuar contra a sobrevivência das empresas de menor tamanho.

No estudo de Sarmento e Nunes (2010) realizado com as empresas da região norte de Portugal, a taxa de rotatividade destas resultou ser a variável mais significativa para explicar a sobrevivência. Em relação à taxa de sobrevivência segundo o tamanho da empresa, foi evidenciado que, à medida que aumenta o tamanho da empresa, a taxa de sobrevivência também aumenta.

Suazo e Pérez (2014), num estudo sobre demografia e sobrevivência de empresas para o Chile no período 2007-2012, apresentaram resultados que servem de parâmetro de comparação devido à dinâmica recente da economia daquele país. Foi observado que, do total das empresas, $83 \%$ sobreviveram ao primeiro ano; $77 \%$, ao segundo ano; e 69\%, ao terceiro ano.

O trabalho de López-Garcia e Puente (2006) sobre demografia e sobrevivência de empresas na Espanha apresentou resultados para as várias especificações do modelo de Cox. Numa especificação não linear em que não existe uma forma predeterminada do modelo, o maior tamanho da empresa do tipo start-up medido em termos do número de empregados afetou de forma positiva a taxa de sobrevivência. Também foi confirmado o efeito positivo da concentração de mercado sobre a sobrevivência.

Em resumo, nos estudos apresentados é perceptível a maior importância outorgada às restrições e circunstâncias que rodeiam a sobrevivência das empresas de menor tamanho. No entanto, é possível regatar alguns dos fatores que afetam indistintamente a permanência das empresas, sejam estas de porte pequeno ou grande: o tamanho do estabelecimento afeta de forma direta e positiva a sobrevivência; o acesso ao mercado de crédito bem como a capacidade de endividamento; a defasagem tecnológica e a possibilidade para gerar inovações; as estruturas de mercado concentradas; a qualificação dos recursos humanos nas empresas; etc.

A disputa por um espaço no mercado, em condições adversas, no caso das menores empresas, foi reconhecida e aceita por Marshall, assim como por Schumpeter, para quem a sobrevivência depende do ritmo em que a pequena empresa consegue introduzir inovações. Audretsch (1991) destaca o estimulo que 
um regime tecnológico específico exerceria em favor do ingresso de pequenas empresas com evidentes tendências à inovação. Mas, mesmo na ausência de tal regime, e em circunstâncias em que prevalecem estruturas produtivas intensivas em capital ou economias de escala, admite-se a possibilidade de que empresas pequenas ingressem em ambientes tecnológicos elevados, não descartando a sua sobrevivência.

Fotopoulos e Louri (2000) assim como Wennberg e Lindqvist (2010), destacaram o espaço geográfico como um dos fatores a afetar a sobrevivência. Os primeiros salientaram o papel das áreas metropolitanas, enquanto incubadoras de negócios. No caso dos segundos, ao investigarem o efeito que as aglomerações de unidades industriais regionais produzem sobre o desempenho e a sobrevivência de empresas, concluíram que tal possibilidade é possível.

\section{Metodologia}

A análise de sobrevivência pode ser entendida como um conjunto de procedimentos cujo objetivo é determinar, principalmente, a probabilidade de sobrevivência e o risco de falha ou fechamento de um grupo de indivíduos, tendo como determinantes o próprio tempo e/ou um conjunto de variáveis denominadas covariáveis. Conforme César (2005), o tempo de sobrevivência está sujeito a variações aleatórias e, como tal, possui uma distribuição aleatória. Tal distribuição pode ser caracterizada pelas seguintes funções: função de sobrevivência, função densidade de probabilidade, função de risco e função de risco acumulada.

O procedimento usado na análise de sobrevivência "permite tratar de problemas nos quais a ocorrência do evento não é constante ao longo do tempo" (CARVALHO et al, 2011, p.78). Os autores interpretam a letra $T$ como o tempo transcorrido até a ocorrência do evento, assim, os eventuais valores de $T$ correspondem aos de uma variável aleatória contínua e positiva, cuja função de densidade de probabilidade é denominada $f(t)$ e é interpretada como a probabilidade de uma empresa sofrer o evento num intervalo infinitesimal:

$$
f(t)=\lim _{\varepsilon \rightarrow 0^{+}} \frac{\operatorname{Pr}(t \leq T<t+\varepsilon)}{\varepsilon}
$$

Já a função de sobrevivência $\mathrm{S}(\mathrm{t})$ é definida como a probabilidade de uma empresa sobreviver por um período igual ou superior a um determinado tempo t.

$$
S(t)=\operatorname{Pr}(T \geq t)
$$


A função de distribuição acumulada $F(t)$ da variável aleatória $T$ é definida como a probabilidade de o evento ocorrer até o tempo $t$.

$$
F(t)=\operatorname{Pr}(T \leq t)
$$

Portanto, a função de sobrevivência também é escrita como o complemento da função de distribuição acumulada:

$$
S(t)=1-\operatorname{Pr}(T \leq t)=1-F(t)
$$

Fica claro que a função de sobrevivência é igual a um no início do período, isto é, $S(0)=1$, e, à medida que o tempo transcorre, $S(t)$ tende a diminuir ou a permanecer constante, mas, se t tende ao infinito, então, $S(\infty)=0$.

Assim como a análise de sobrevivência refere-se à probabilidade de sobreviver após um determinado tempo, quando a questão se inverte, ela é formulada em termos do risco. Isto é, indaga-se a respeito de qual o risco de um estabelecimento fechar ou morrer após ter sobrevivido por um determinado período de tempo. A função risco, expressa pelo símbolo $\lambda(t)$, é definida "como o risco instantâneo de um estabelecimento sofrer o evento entre o tempo $t \mathrm{e}(t+\varepsilon)$ dado que ele sobreviveu até o tempo t." (CARVALHO et al, 2011, p. 83).

$$
\lambda(t)=\lim _{\varepsilon \rightarrow 0} \frac{\operatorname{Pr}[t \leq T<t+\varepsilon) \mid T \geq t]}{\varepsilon}
$$

A função risco pode apresentar diversos formatos geométricos em relação ao tempo: uma reta constante, decrescente, crescente, crescente e decrescente, decrescente e crescente, etc. Por outro lado, é possível determinar o risco de um estabelecimento decretar o seu encerramento no primeiro ano, segundo ano, etc., o que é realizado por meio da função risco acumulado. Em termos matemáticos, ela é obtida a partir da "soma de todos os riscos em todos os tempos até o tempo $t$ " (CARVALHO et al.,2011, p. 88)

$$
H(t)=\int_{0}^{t} \lambda(t) d t
$$

Os autores supracitados apontam que o risco acumulado é uma taxa não restrita ao intervalo $[0,1]$ e que pode ser escrita em termos da função de sobrevivência:

$$
H(t)=-\ln (S(t))
$$

Esses autores fazem notar que as funções expostas anteriormente se encontram relacionados entre si; por exemplo, quando se estima a função sobrevivência, também podem ser obtidas as funções do risco e do risco acumulado. 
Finalmente, é apontado que a estimação das funções acima é realizada usando o método da máxima verossimilhança. (CARVALHO et al, 2011, p. 92).

Assim, a análise de sobrevivência propõe uma série de modelos para avaliar a evolução da taxa de mortalidade em função do tempo. Porém, não somente para a relação entre a taxa de mortalidade e o tempo, mas também para a relação entre aquela e as covariáveis registradas para os indivíduos. Os procedimentos que atendem a essas condições são os modelos Kaplan-Meier ${ }^{7}$ e Cox $^{8}$.

A abordagem estatística da análise de sobrevivência refere-se basicamente a três tipos de modelos: não-paramétricos, paramétricos e semiparamétricos. Esses modelos têm sido invariavelmente utilizados nas pesquisas sobre sobrevivência, como mostram Fuentelsaz, Gomez e Polo (2004) e Kleinbaum e Klein (2012). Na abordagem não-paramétrica, não existe qualquer suposição a respeito da distribuição probabilística do tempo de sobrevivência, $T$, e, portanto, não se estimam os seus parâmetros.

Carvalho et al. (2011) denominam os tempos $t_{1}<t_{2}<t_{3}<\ldots<t_{m}$ como os $\mathrm{m}$ momentos em que ocorrem os eventos numa amostra de $\mathrm{n}$ estabelecimentos. Chamando de $\mathrm{R}\left(\mathrm{t}_{\mathrm{j}}\right)$ o número de estabelecimentos em risco no momento $t_{j} \mathrm{e}$ de $\Delta N\left(t_{j}\right)$ o número de eventos ocorridos em $\mathrm{t}_{\mathrm{j}}$, a probabilidade de sobrevivência no momento $t_{j}$, segundo Kaplan-Meier, é estimada pelo número de sobreviventes nesse momento, $\left[R\left(t_{j}\right)-\Delta N\left(t_{j}\right)\right]$, dividido pelo número de estabelecimentos em situação de risco até aquele momento $R\left(t_{j}\right)$

A probabilidade de sobrevivência de Kaplan-Meier é:

$$
\hat{S}_{k m}(t)=\prod_{j: t_{j} \leq t} \frac{R\left(t_{j}\right)-\Delta N\left(t_{j}\right)}{R\left(t_{j}\right)}=\prod_{j: t_{j} \leq t} 1-\frac{\Delta N\left(t_{j}\right)}{R\left(t_{j}\right)}
$$

A representação gráfica da expressão $S_{k m}(t)$ acima é decrescente em relação ao tempo e permite a geração de outras funções que fazem parte da análise de sobrevivência, a exemplo da denominada função risco acumulado, $H_{k m}(t)=-\ln (S(t))$. Alternativamente, é possível estimar a função risco acumulado utilizando o estimador de Nelson-Aalen, expresso da seguinte maneira:

\footnotetext{
${ }^{7}$ Edward L Kaplan (1920-2006), matemático, e Paul Meier (1924-2011), estatístico, foram dois pesquisadores estadunidenses que desenvolveram o estimador não paramétrico de Kaplan-Meier, utilizado para mensurar a quantidade de pacientes que sobrevivem a um tratamento médico. A publicação de destaque dos autores foi: "Nonparametric estimation from incomplete observations", no Journal of the American Statistical Association de 1958.

${ }^{8}$ David Cox (1924- ), estatístico inglês reconhecido pela regressão de Cox, utilizada na análise de sobrevivência.
} 


$$
\hat{H}_{n a}(t)=\sum_{j: t_{j} \leq t} \frac{\Delta N\left(t_{j}\right)}{R\left(t_{j}\right)}
$$

Adicionalmente, a partir da expressão acima, pode-se obter outra estimativa não-paramétrica para a sobrevivência, que é dada por $\hat{S}_{n a}(t)=\exp \left(-\hat{H}_{n a}(t)\right)$.

Por outro lado, é possível obter a representação da função de sobrevivência de Kaplan-Meier segundo estratos originados na classificação de cada uma das covariáveis. Dessa maneira, pode-se expressar a sobrevivência de um subgrupo de estabelecimentos, que deveria ser, em tese, diferente do comportamento de outro subgrupo.

Para comparar a representação dessas funções de risco (sobrevivência) e verificar a sua diferença por subgrupos, recorre-se a testes de hipóteses, entre os quais, se destaca o log-rank. Este teste "compara os valores observados e esperados de cada estrato sob a hipótese nula de que o risco seja o mesmo em todos os grupos." (CARVALHO, et al., 2011, p.121). Ou seja, o que se indaga é se a incidência de um evento é igual em cada estrato (num total de k estratos), portanto:

$$
H_{0}: \lambda_{1}(t)=\lambda_{2}(t)=\ldots .=\lambda_{k}(t)
$$

O teste log-rank “... compara a distribuição da ocorrência dos eventos observados em cada estrato com a distribuição que seria esperada se a incidência fosse igual em todos os estratos" (CARVALHO et al., 2011, p.121). Em termos operacionais, primeiramente se procede à estimativa do número de eventos esperados para cada estrato $E_{k}(t)$ segundo a hipótese nula. Depois, é calculada a estatística do teste que segue uma distribuição qui-quadrado com $k$-1 graus de liberdade, quando a hipótese nula é verdadeira. Formalmente, é realizado a partir dos seguintes cálculos:

$$
\begin{aligned}
& E_{k}(t)=\Delta N(t) \frac{R_{k}(t)}{R(t)} \\
& \log -\operatorname{Rank}=\frac{\left(O_{1}-E_{1}\right)^{2}}{\operatorname{Var}\left(O_{1}-E_{1}\right)}
\end{aligned}
$$

onde: $E_{1}$ é o número de eventos esperados no estrato 1 , e $O_{1}$ é o número de eventos observados no estrato 1. O critério de decisão consiste em rejeitar a hipótese nula, para um $p$-valor menor que $5 \%$.

A esse respeito, Favero (2014) comenta que é possível efetuar comparações entre grupos mediante os estimadores de Kaplan-Meier e calcular a significância dos resultados com base no teste de log-rank. Contudo, "quando existir uma série de 
variáveis explanatórias e, em particular, quando algumas destas variáveis forem contínuas, é muito mais útil que se utilizem métodos de regressão, como a regressão de riscos proporcionais, também conhecida por regressão de Cox" (FAVERO, 2014, p.211).

Assim, quando o estudo está centrado não em estimar os parâmetros da distribuição de probabilidade do tempo de sobrevivência, senão em estimar o efeito de covariáveis, então, a referência deve ser feita ao modelo de Cox, o qual ajusta o risco em função do risco baseline, $\lambda_{o}(t)$, e de um vetor de covariáveis conforme a seguinte expressão:

$$
\lambda(t \mid x)=\lambda_{0}(t) \exp \left(x_{1} \beta_{1}+x_{2} \beta_{2}+\ldots+x_{p} \beta_{p}\right)
$$

Cabe mencionar de acordo com Molinero (2001) que a função baseline $\lambda_{0}(t)$ não é de importância fundamental no modelo de Cox uma vez que objetivo deste é estimar o impacto das covariáveis sobre a sobrevivência. "Para la estimación de un modelo de riesgos proporcionales se podría postular para la función de riego basal un modelo matemático cualquiera, por ejemplo el de Weibull. Sin embargo, la aproximación propuesta por Cox se basa en que a menudo no se conoce la forma de $\lambda_{0}(t)$ y además no es de interés primordial, ya que el verdadero objetivo es valorar la influencia de los factores pronóstico en la supervivencia. Por ello en el modelo de Cox no se determina $\lambda_{0}(t)$ " (MOLINERO, LUIS. 2001, p.1)

Expressando a relação do risco do evento para duas entidades $k$ e $/$ em função das covariáveis $x_{k}$ e $x_{l}$, temos como resultado uma constante que não depende do tempo, isto é:

$$
\frac{\lambda_{k}\left(t \mid x_{k}\right)}{\lambda_{l}\left(t \mid x_{l}\right)}=\frac{\exp \left(x_{k} \beta\right)}{\exp \left(x_{l} \beta\right)}=\exp \left(x_{k} \beta-x_{l} \beta\right)
$$

O que se pretende mostrar com a expressão acima é que o risco de fechamento de uma entidade não varia ao longo do tempo em relação à outra entidade, razão pela qual se denomina modelo de riscos proporcionais.

Então, assumindo-se a proporcionalidade conforme a descrição realizada, podem-se estimar os efeitos das covariáveis sem que seja realizada nenhuma suposição sobre a distribuição do tempo de sobrevivência, uma condição dos modelos paramétricos. O modelo de Cox não precisa assumir "qualquer distribuição estatística para a função de risco basal $\lambda_{0}(t)$. Assume apenas que as covariáveis agem multiplicativamente sobre o risco, sendo esta a parte paramétrica do modelo". (CARVALHO et al, 2011, p.186) 
O banco de dados utilizado corresponde aos microdados identificados dos estabelecimentos formais do Rio Grande do Sul referentes à Relação Anual de Informações Sociais (RAIS) do Ministério do Trabalho e Emprego. Em função da disponibilidade dos microdados identificados da RAIS, o período analisado vai de 2007 a 2013. Consideram-se as informações dos estabelecimentos cujo tamanho, em termos do número de pessoas ocupadas, é superior a um trabalhador. O recorte temporal nos dados decorre da sua disponibilidade, assim como das covariáveis utilizadas: o tamanho do estabelecimento, a localização em termos das mesorregiões e a atividade econômica em termos da sua classificação na CNAE 2.0.

O tamanho foi classificado segundo as seguintes faixas correspondentes ao número de pessoas ocupadas: a) de uma a quatro pessoas (Tamanho 1); b) de cinco a nove pessoas (Tamanho 2); c) de 10 a 19 pessoas (Tamanho 3); d) de 20 a 49 pessoas (Tamanho 4); e, e) acima de 50 pessoas (Tamanho 5).

Da mesma maneira, a atividade foi distribuída em sete categorias: a) agricultura e indústrias extrativas, representando o setor primário (Agric); b) indústria de transformação e energia (Ind); c) construção (Constr); d) comércio, transporte, alojamento, alimentação, informação e comunicação (Com); e) atividade financeira, imobiliária e de seguros (Fin); f) administração pública (Adm); g) educação, saúde, arte, cultura e outros serviços (Educ).

Finalmente, em relação à distribuição espacial, os estabelecimentos foram distribuídos nas sete mesorregiões do Rio Grande do Sul, conforme classificação do IBGE: a) Noroeste Rio-grandense (Noroeste); b) Nordeste Rio-grandense (Nordeste); c) Centro Ocidental Rio-grandense (CenOci); d) Centro Oriental Riograndense (CenOri); e) Metropolitana de Porto Alegre (Metro); f) Sudoeste Riograndense (Sudoeste); e g) Sudeste Rio-grandense (Sudeste).

\section{Resultados e discussão}

Esta seção está dividida em duas partes. A primeira apresenta as evidências da sobrevivência das empresas obtidas a partir do método de Kaplan-Meier, segundo o tamanho das empresas, o tipo de atividade e a localização geográfica. A segunda parte apresenta o resultado do modelo de Cox, mediante o qual se avalia 0 efeito do tamanho do estabelecimento sobre o risco de fechamento dos estabelecimentos. De acordo com a literatura, espera-se que o tamanho da empresa, definido em termos do número de pessoas ocupadas, seja inversamente relacionado com o risco de fechamento. 
A análise de sobrevivência verificada por meio do procedimento de KaplanMeier é realizada com os estabelecimentos nascidos em 2007. O seu acompanhamento temporal termina no ano de 2013. Assim, é possível obter a informação da sobrevivência das empresas até seu sexto ano de vida ativa. As informações das observações que ultrapassam o final do período são consideradas censuradas. Estas informações correspondem às empresas que continuam vivas após o ano de 2013 e, portanto, não se sabe o seu tempo de sobrevivência.

No Quadro 01 observam-se a função de sobrevivência de todos os estabelecimentos da forma como foi proposta no parágrafo acima, bem como os valores das respectivas taxas de sobrevivência. Encontra-se, por exemplo, que $86,11 \%$ dos estabelecimentos recém-nascidos no ano de 2007 sobreviveram ao primeiro ano de vida; $70,37 \%$ dos estabelecimentos sobreviveram ao segundo ano, e assim sucessivamente até o último ano disponível, sendo que no sexto ano apenas 35,74\% das empresas nascidas em 2007 continuavam vivas. Também pode ser verificado que metade das empresas que iniciaram as suas atividades no ano de 2007 permaneceram funcionando entre quatro e cinco anos.

Quadro 01 - Curva de sobrevivência de Kaplan-Meier, Rio Grande do Sul, 2007-2013.

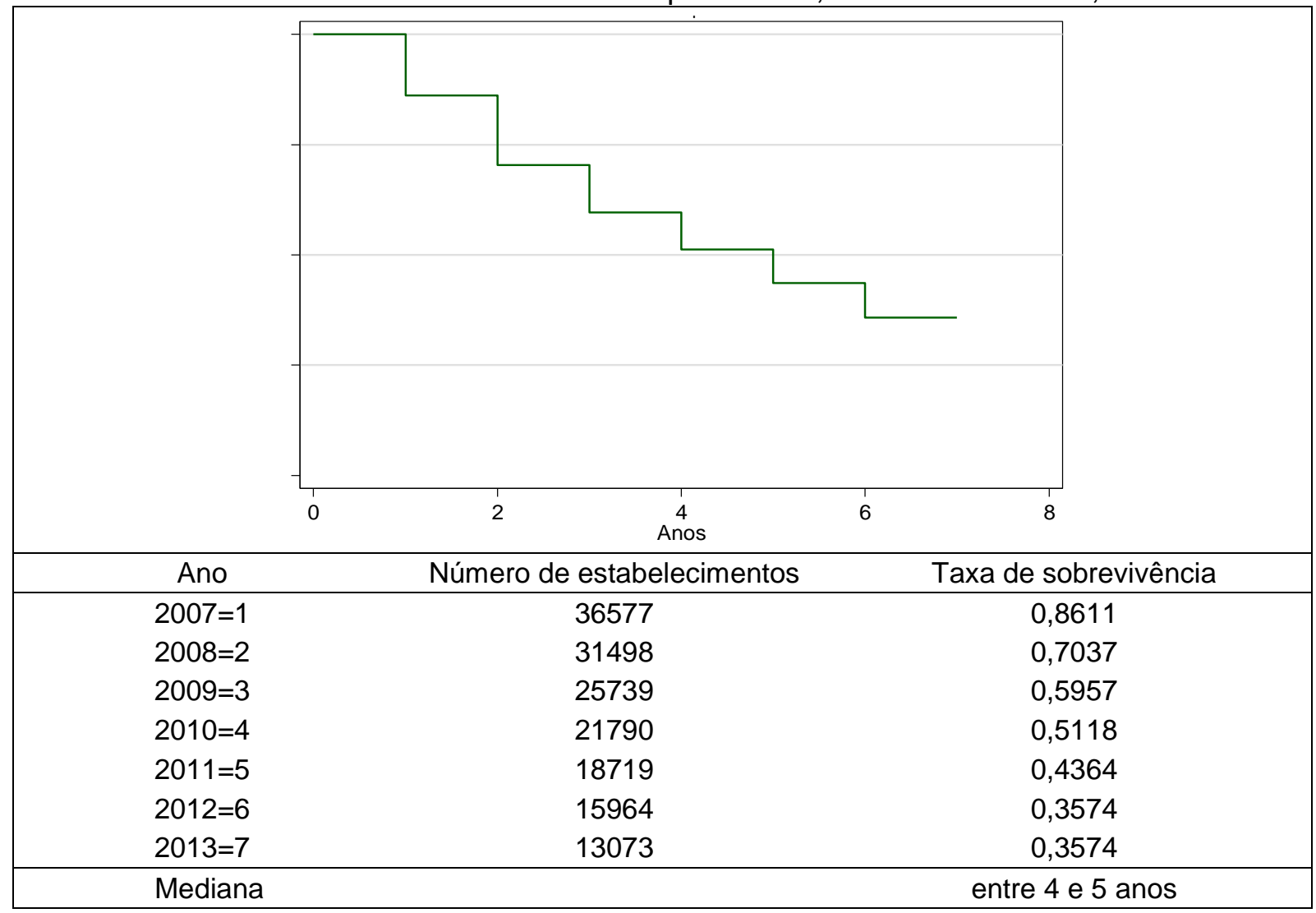

Fonte: Elaboração própria a partir dos resultados da pesquisa.

Nota: a mediana refere-se ao tempo de vida até a morte de 50\% das empresas do grupo. 
Classificando-se os estabelecimentos por estratos de tamanho, encontraramse diferentes funções de sobrevivência para os cinco tamanhos preestabelecidos, tal qual se observa no Quadro 2. O teste de hipóteses para a sobrevivência por grupos de tamanho levantou como hipótese nula a igualdade, por estratos de tamanho, da probabilidade de falência ou morte. Assim, o resultado encontrado para o estatístico Chi2 resultou igual a 264,95 com $\operatorname{Pr}>\operatorname{chi} 2=0,0000$, portanto, menor que $5 \%$, o que permitiu rejeitar a hipótese nula, ou seja, a diferença por estratos segundo 0 tamanho resultou ser estatisticamente significativa.

Assim, os estabelecimentos de menor tamanho, na faixa entre um a quatro pessoas ocupadas (Tamanho1), apresentaram a menor sobrevivência entre todas as faixas. No primeiro ano, sobreviveram $85,39 \%$ do total, e, no sexto ano, sobreviveram apenas $33,82 \%$. Já os estabelecimentos de maior tamanho, correspondentes às faixas entre 20 e 49 pessoas ocupadas (Tamanho 4) e à faixa acima de 50 pessoas ocupadas (Tamanho 5), apresentaram as maiores probabilidades de sobrevivência: $90,45 \%$ e $88,42 \%$ respectivamente no primeiro ano; e $46,11 \%$ e $45,76 \%$ no sexto ano.

A metade de todos os estabelecimentos que iniciaram as suas atividades em 2007 correspondentes ao menor tamanho, isto é, à faixa entre uma e quatro pessoas ocupadas, sobreviveram apenas entre o terceiro e quarto ano de vida. Entre os estabelecimentos com mais de cinco pessoas ocupadas, metade sobreviveram entre cinco a seis anos de vida. Ou seja, em relação aos estabelecimentos de maior tamanho, a vida ativa dos estabelecimentos de menor tamanho é menor.

A relação positiva entre 0 tamanho do estabelecimento e a taxa de sobrevivência verificada no Quadro 02 é coerente com o resultado encontrado em Resende et al. (2016), para quem tal associação responde à eficiência de escala. Outros motivos para tal relação comportamental podem estar relacionados com o fato de as empresas de maior tamanho apresentar maior intensidade de capital especializado, as barreiras à entrada e à saída, acesso privilegiado ao mercado de crédito, e contarem com departamentos dedicados à pesquisa e à inovação. 
Quadro 02 - Curva de sobrevivência de Kaplan-Meier segundo o tamanho dos estabelecimentos, Rio Grande do Sul, 2007-2013.

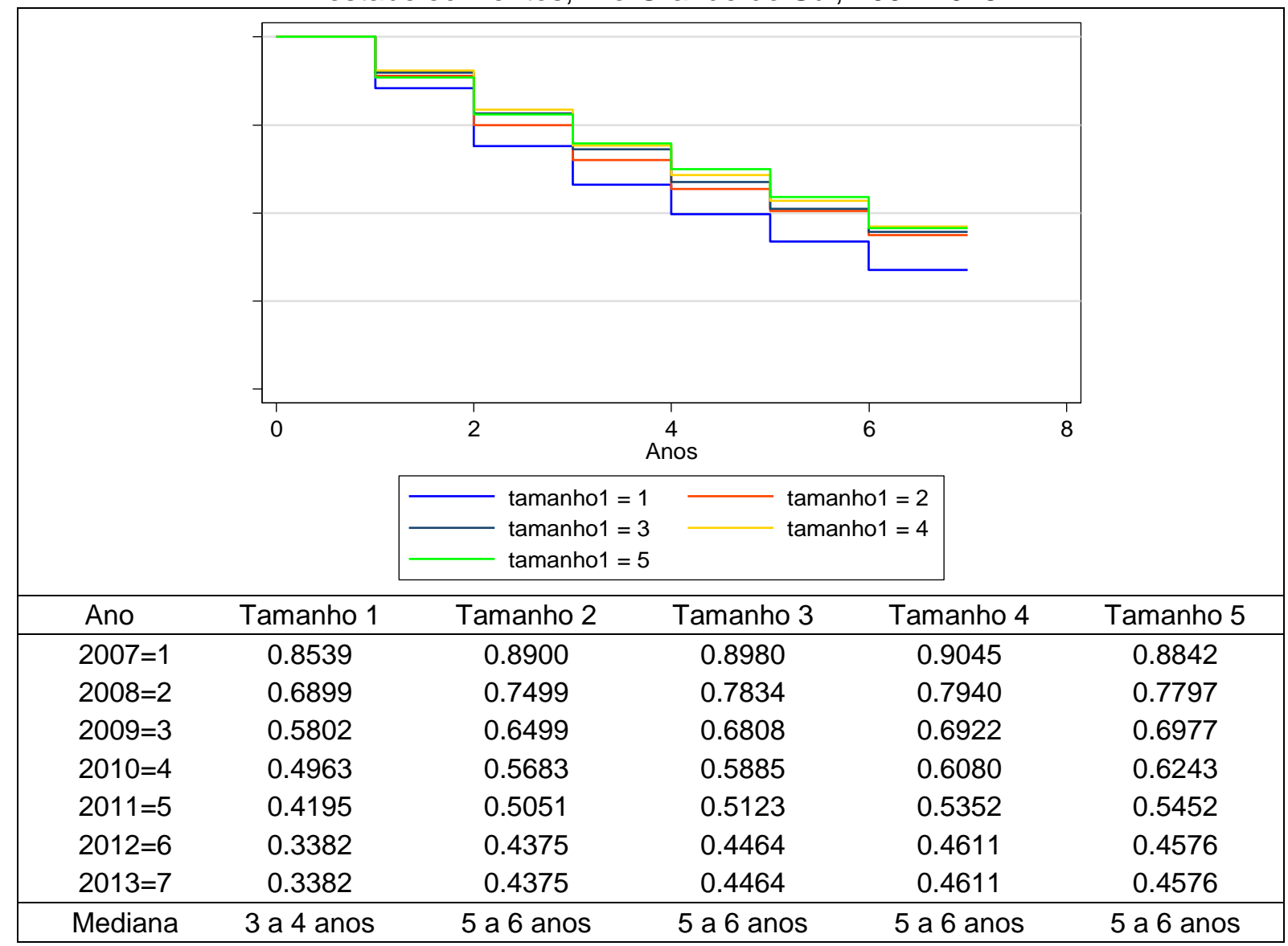

Fonte: Elaboração própria a partir dos resultados da pesquisa.

Nota 1: a mediana refere-se ao tempo de vida até a morte de $50 \%$ das empresas do grupo.

Nota 2: Tamanho 1: de 1 até 4 pessoas ocupadas; Tamanho 2: de 5 até 9 pessoas ocupadas; Tamanho 3: de 10 até 19 pessoas ocupadas; Tamanho 4: de 20 até 49 pessoas ocupadas; e Tamanho 5: acima de 50 pessoas ocupadas.

No estudo de Resende et al. (2016), referente ao período de 1996-2005, as empresas pequenas e médias do Brasil apresentaram para os primeiros quatro anos de vida as seguintes taxas de sobrevivência: $74,74 \%$; $59,93 \%$; 49,93\%; e 42,22\%, respectivamente. Os valores obtidos neste ensaio para o mesmo tamanho (Tamanhos 1 a 4, do Quadro 2) correspondentes ao Rio Grande do Sul foram: 88,66\%; 75,43\%; 65,08\%; e, 56,53\%. Assim, desconsiderando as diferenças entre os períodos analisados nos dois estudos, constata-se que as taxas de sobrevivência de empresas gaúchas foram superiores às de respectivo tamanho no Brasil.

Os resultados da sobrevivência segundo a atividade econômica estão no Quadro 03. O respectivo teste de log-rank resultou num estatístico chi2 igual a 1132,65 com Pr>chi2 = 0,0000; portanto, para um nível de significância de 5\%, rejeitou-se a hipótese nula em favor da hipótese alternativa, isto é, a diferença entre estratos por atividade econômica é estatisticamente significativa. 
Conforme se pode verificar pela mediana, a metade dos estabelecimentos do setor da construção sobreviveu somente até o segundo ano de vida, denotando a maior fragilidade entre todos os setores da atividade econômica. Na sequência se encontram os estabelecimentos da educação, saúde, arte, cultura e outros serviços, metade deles sobreviveu entre três e quatro anos. No caso das empresas da atividade financeira e correlatas, metade delas pôde sobreviver entre cinco e seis anos, constituindo-se assim na atividade privada de maior longevidade.

Quadro 03 - Curva de sobrevivência de Kaplan-Meier segundo o setor se atividade dos estabelecimentos, Rio Grande do Sul, 2007-2013.

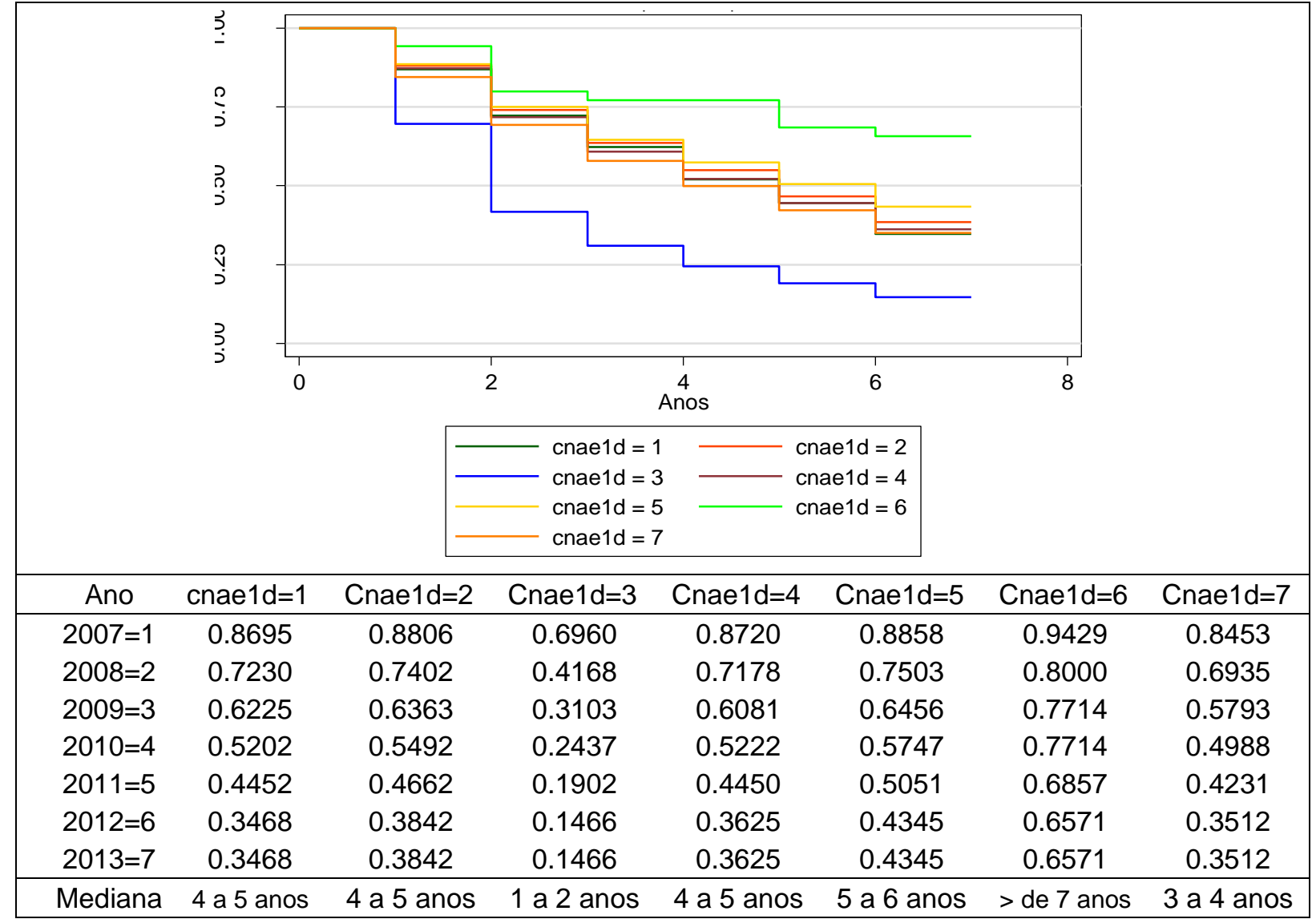

Fonte: Elaboração própria a partir dos resultados da pesquisa.

Nota 1: a mediana refere-se ao tempo de vida até a morte de $50 \%$ das empresas do grupo.

Nota 2: Grupos de setores segundo o CNAE2. Número 1: Agricultura e Indústria Extrativa; Número 2: Indústria de Transformação e Energia; Número 3: Construção; Número 4: Comércio, Reparação de Veículos, Transporte, Alojamento e Alimentação, Informação e Comunicação; Número 5: Atividade Financeira, Seguros, Negócios Imobiliários, Serviços Profissionais; Número 6: Administração Pública; Número 7: Educação, Saúde, Arte, Cultura, Outros Serviços.

Conforme destacado na revisão da bibliografia, as atividades industriais e financeiras ao apresentarem maior potencial na geração de economias de escala e, no caso específico da indústria, por esta ser uma atividade capital intensiva e apresentar maior produtividade do trabalho, são também as mais longevas. Já as atividades de educação, saúde e artes, bem como as de comércio e serviços, nas quais o trabalho é uma atividade fim, a possibilidade de apresentarem economias de 
escala é menor; portanto, são atividades cuja sobrevivência também é menor. No caso específico da atividade da construção, que apresenta a menor sobrevivência, esta pode ser explicada pela maior taxa de rotatividade, tal como aponta a literatura revisada.

Finalmente, pode ser observado no Quadro 4 a sobrevivência das empresas segundo a sua localização geográfica. O resultado do teste de hipóteses apontou para um estatístico chi2 igual a 97,90 com um $\operatorname{Pr}>\operatorname{chi} 2=0,0000$, o que permitiu que fosse rejeitada a hipótese nula com um nível de significância de $5 \%$. Isto é, a diferença de probabilidade de sobrevivência segundo a localização geográfica dos estabelecimentos é estatisticamente significativa.

Analisando os resultados do Quadro 04 é possível verificar que os estabelecimentos da mesorregião centro-ocidental (mesors=3) apresentaram a menor sobrevivência, enquanto que os estabelecimentos da região nordeste (mesors=2) foram os que apresentaram a maior sobrevivência. De acordo com os valores da mediana, metade dos estabelecimentos da mesorregião Centro-Ocidental e os da mesorregião Metropolitana de Porto Alegre sobreviveram entre três e quatro anos, sendo, portanto, as regiões mais frágeis nesse quesito. No restante das mesorregiões, a sobrevivência dos estabelecimentos melhora um pouco, uma vez que a metade deles nas respectivas regiões teve uma longevidade entre quatro a cinco anos.

Quadro 04 - Curva de sobrevivência de Kaplan-Meier segundo a localização dos estabelecimentos, Rio Grande do Sul, 2007-2013.

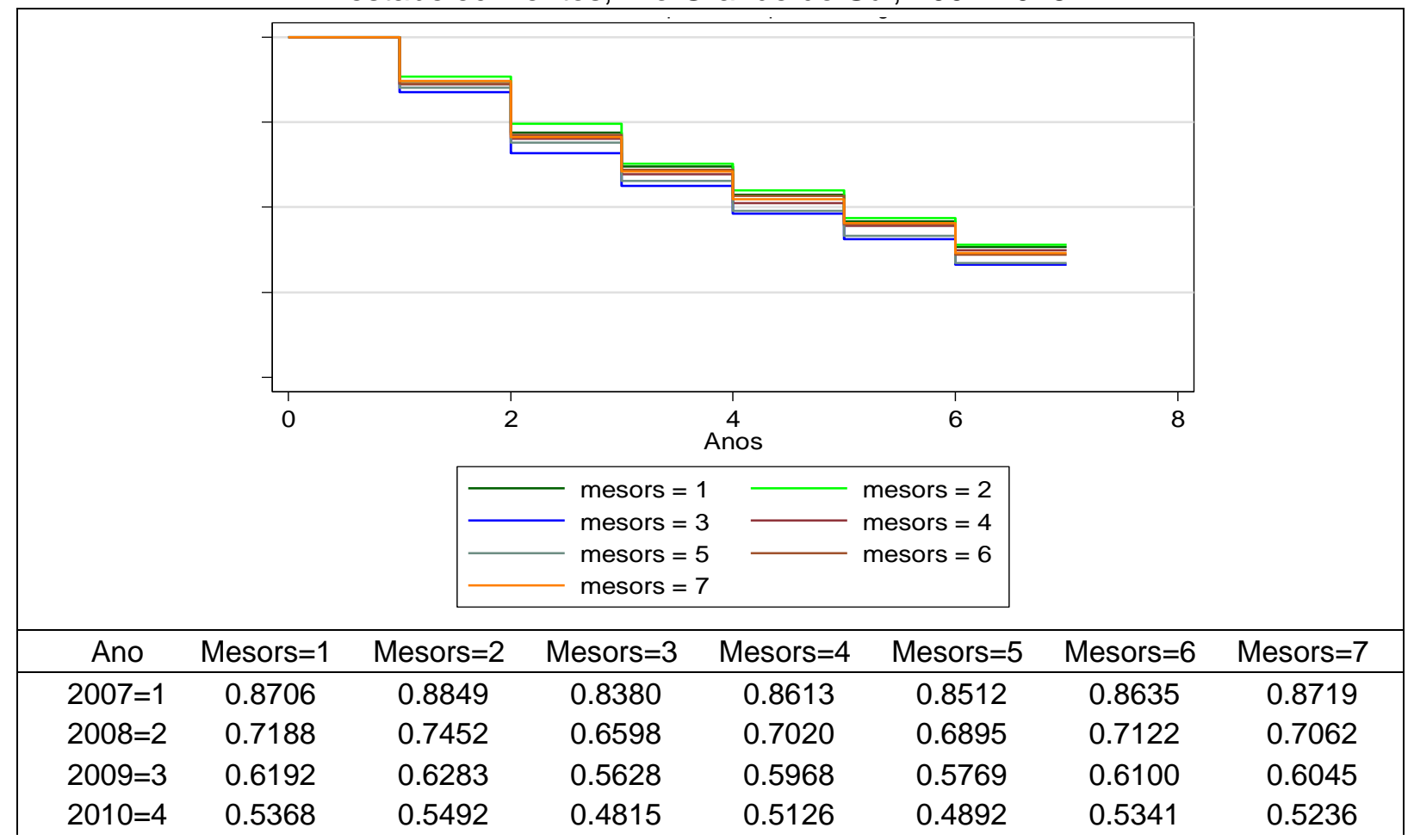




\begin{tabular}{|cccccccc|}
$2011=5$ & 0.4588 & 0.4674 & 0.4053 & 0.4446 & 0.4162 & 0.4505 & 0.4525 \\
$2012=6$ & 0.3837 & 0.3892 & 0.3307 & 0.3735 & 0.3362 & 0.3597 & 0.3657 \\
$2013=7$ & 0.3837 & 0.3892 & 0.3307 & 0.3735 & 0.3362 & 0.3597 & 0.3657 \\
\hline Mediana & 4 a 5 anos & 4 a 5 anos & 3 a 4 anos & 4 a 5 anos & 3 a 4 anos & 4 a 5 anos & 4 a 5 anos \\
\hline
\end{tabular}

Fonte: Elaboração própria a partir dos resultados da pesquisa.

Nota 1: a mediana refere-se ao tempo de vida até a morte de $50 \%$ das empresas do grupo.

Nota 2: Mesorregiões do Rio Grande do Sul: Meso 1: Noroeste Rio-grandense; Meso 2: Nordeste Riograndense; Meso 3: Centro-Ocidental Rio-grandense; Meso 4: Centro-Oriental Rio-grandense; Meso 5: Metropolitana de Porto Alegre; Meso 6: Sudoeste Rio-grandense; Meso 7: Sudeste Rio-grandense.

Embora a representação gráfica das funções de sobrevivência e os resultados das taxas de sobrevivência sejam bastante parecidos entre as mesorregiões, o Nordeste Rio-grandense resulta ser o espaço geográfico onde os estabelecimentos sobrevivem por mais tempo. Essa situação pode ser explicada, em tese, pelo fato de esse espaço hospedar um maior número de aglomerações produtivas industriais e contar com externalidades positivas que acabam fortalecendo a produtividade e a competitividade dos estabelecimentos, vitais para o alongamento da vida produtiva das empresas. Além disso, ao ser uma mesorregião predominantemente industrial, está constituída preferencialmente por empresas intensivas em capital, com economias de escala e maior escala eficiente mínima. No outro extremo, situa-se a mesorregião Centro-Ocidental Rio-grandense, caracterizada por uma economia centrada na agricultura e nos serviços, os quais, diferentemente da atividade industrial, tendem a apresentar uma menor sobrevivência dos seus estabelecimentos.

Diferentemente do procedimento de Kaplan-Meier, em que a variável tempo é determinante da sobrevivência e/ou do risco de morte dos estabelecimentos, no modelo de Cox o risco de fechamento é o resultado do efeito de uma ou mais variáveis explicativas. A escolha destas variáveis deve ser feita à luz da literatura pertinente e em função da disponibilidade de informações. As informações individuais por empresa, presentes na base de dados, que foram testadas para explicar a sobrevivência das empresas são o tamanho da empresa, o setor em que a empresa opera e a região em que a empresa está instalada.

Para usar o modelo de Cox as variáveis devem passar no teste de proporcionalidade de risco. Das três variáveis testadas, apenas o tamanho das empresas passou no teste. A Tabela 01 apresenta o teste de proporcionalidade de risco, cuja hipótese nula admite a proporcionalidade dos riscos entre os estabelecimentos à medida que tempo aumenta. Conforme evidenciado, para um nível de significância de 5\%, é aceita a hipótese nula, validando os resultados da variável Tamanho do estabelecimento sobre o risco de morte. 
Tabela 01 - Teste de proporcionalidade do risco na regressão de Cox.

\begin{tabular}{l|c|c|c}
\hline Variáveis & Chi2 & df & Prob>chi2 \\
\hline Tamanho dos estabelecimentos & 5,62 & 4 & 0,2294 \\
Atividade dos estabelecimentos & 83,26 & 6 & 0,0000 \\
Localização geográfica dos estabelecimentos & 14,22 & 6 & 0,0273 \\
\hline
\end{tabular}

Fonte: Elaboração própria a partir dos resultados da pesquisa.

O empreendedor, ao escolher um determinado tamanho para a sua empresa, provavelmente avalia de forma explícita ou implícita as expectativas em relação ao lucro e às vendas, à presença de externalidades, às barreiras à entrada e à saída, etc. Tomando como variável independente o tamanho do estabelecimento, o resultado da regressão de Cox é apresentado na Tabela 02.

Tabela 02 - Resultado do modelo de Cox (variável explicativa: Tamanho do estabelecimento).

\begin{tabular}{c|c|c|c|c|cc}
\hline Tamanho & Haz. Ratio & Std. Err. & $\mathrm{Z}$ & $\mathrm{P}>\mathrm{Z}$ & [95\% Conf. & Interval] \\
\hline 2 & 0.785473 & 0.017967 & -10.56 & 0.000 & 0.751036 & 0.821490 \\
3 & 0.756159 & 0.024917 & -8.48 & 0.000 & 0.708865 & 0.806609 \\
4 & 0.724283 & 0.035347 & -6.61 & 0.000 & 0.658213 & 0.796985 \\
5 & 0.729822 & 0.052927 & -4.34 & 0.000 & 0.633122 & 0.841292 \\
\hline
\end{tabular}

Log likelihood $=-238761 \quad$ LRchi2 $(4)=235,77 \quad$ Prob $>$ chi2 $=0,0000$

Fonte: Elaboração própria a partir dos resultados da pesquisa

Nota: Tamanho dos estabelecimentos por pessoas ocupadas: Tamanho 1: de 1 até 4 pessoas ocupadas; Tamanho 2: de 5 até 9 pessoas ocupadas; Tamanho 3: de 10 até 19 pessoas ocupadas; Tamanho 4: de 20 até 49 pessoas ocupadas; e Tamanho 5: acima de 50 pessoas ocupadas.

A leitura de cada coeficiente toma como referência os estabelecimentos de menor tamanho (Tamanho 1). Constata-se na Tabela 02, que os sinais dos coeficientes são coerentes com o efeito esperado, isto é, o menor tamanho dos estabelecimentos apresenta uma taxa de morte maior que a de tamanhos maiores. Assim, por exemplo, os estabelecimentos cujo tamanho está identificado com o número 2 (Tamanho 2), apresentaram uma probabilidade de morrer inferior em $21,46 \%$ (1 - 0,785473) em relação aos estabelecimentos de referência.

\section{Considerações finais}

Este estudo teve por objetivo medir a taxa de sobrevivência das empresas entrantes do Rio Grande do Sul ao longo do período de 2007-2013 e avaliar possíveis fatores que explicam esta sobrevivência. Para isso, inicialmente foi aplicado o procedimento de Kaplan-Meier de forma a obter as funções de sobrevivência dos estabelecimentos segundo o tamanho, a localização e a atividade 
produtiva. A seguir, por meio do modelo de Cox, estimou-se uma regressão para averiguar os seus possíveis fatores explicativos.

A taxa de sobrevivência das empresas do Rio Grande do Sul nascidas em 2007 e com dois anos de atividade (período considerado crítico para consolidar as atividades produtivas) ficou em $70,37 \%$. A respectiva taxa resultou maior que a registrada por países mais desenvolvidos como: Espanha, 68,7\%; Itália, 68,2\%; e, Finlândia, 64,4\%, mas foi menor que as empresas do Canadá, 76,4\%, e Estados Unidos, 75,5\%. Comparando com respectiva taxa de sobrevivência das empresas do Brasil constituídas em 2007, cujo resultado ficou em 75,6\% (SEBRAE, 2013, p.19), a taxa de sobrevivência das empresas gaúchas ficou atrás.

O resultado da taxa de longevidade das empresas Rio-grandenses segundo o seu tamanho esteve de acordo com o que a literatura e estudos semelhantes descrevem, isto é, as empresas de menor tamanho confirmaram a sua fragilidade dada a menor longevidade apresentada, enquanto que as de maior tamanho tiveram uma maior sobrevivência. A falta de experiência gerencial, a menor inovação e, até a dificuldade de competir num mercado dominado pelas empresas de maior porte pode explicar o desempenho das menores.

Algo parecido pode se dizer a respeito da longevidade das empresas avaliadas segundo o tipo de atividade econômica, as pertencentes ao setor da construção apresentaram a menor taxa de sobrevivência enquanto que as empresas do setor financeiro e da indústria tiveram melhor desempenho. É provável que a maior longevidade nesses setores tenha sido produto de uma estrutura de mercado menos competitiva, com maiores investimentos em capital, qualificação e inovação, e, provavelmente, formados por empresas de maior tamanho.

Em relação à sobrevivência de empresas em nível espacial, os estabelecimentos da mesorregião do Nordeste Rio-grandense apresentaram os melhores indicadores, sendo seguidos de perto pelas empresas da mesorregião do Noroeste Rio-grandense. Provavelmente tenha influenciado na maior longevidade ali detectada, a presença de aglomerações produtivas e as externalidades decorrentes, assim como, a concentração de uma população próxima a um milhão de habitantes, o que reflete a dimensão do seu mercado e a oferta de mão de obra mais especializada.

O modelo de Cox apresentado foi construído com a finalidade de explicar de que maneira as decisões assumidas pelos empreendedores em relação à localização do empreendimento, tamanho da empresa e atividade escolhidas, 
puderam influenciar a sobrevivência dos empreendimentos. Ao ser cada uma dessas variáveis definida em termos de estratos, e ao exigir o modelo de Cox o cumprimento da condição de proporcionalidade de risco, apenas o tamanho da empresa pode informar a respeito da sua influência sobre a sobrevivência. Ficou confirmado que quanto maior o tamanho de uma empresa maior é a sobrevivência dos empreendimentos, e, também, foi determinada a dimensão do efeito do tamanho na sobrevivência.

Espera-se que os resultados apresentados neste ensaio possam contribuir para que os gestores públicos e privados descubram ou evidenciem as fragilidades e fortalezas relacionadas à longevidade das empresas gaúchas quando ela é avaliada segundo o tamanho, o tipo de atividade e a sua localização. Especial atenção deveria ser outorgada aos empreendimentos de menor tamanho, aos do setor da construção e aos localizados na mesorregião Centro-ocidental Rio-grandense uma vez que neles foram obtidos os indicadores mais frágeis de sobrevivência. Provavelmente o estimulo para o aumento da competitividade através da formação de polos de desenvolvimento e/ou aglomerações produtivas seja o caminho para elevar os respectivos indicadores em direção de um patamar mais elevado.

A pesquisa esteve restrita aos dados disponíveis e únicos de cada uma das empresas gaúchas obtidas junto aos microdados da RAIS. Acredita-se que a possibilidade de se contar com dados relacionados com o endividamento, receita, crédito, despesas com inovação e qualificação dos recursos humanos, poderiam melhor sinalizar o comportamento e as causas da maior ou menor sobrevivência das empresas gaúchas. Por este motivo, futuros esforços para encontrar melhores respostas provavelmente requereram desse tipo de informação.

\section{REFERÊNCIAS}

AUDRETSCH, D. New-firm survival and the technological regime. The Review of Economics and Statistics, 73, N. 3, p.441-450, 1991.

AUDRETSCH, D.; MAHMOOD, T. The rate of hazard confronting new firm and plants in US manufacturing. Review of Industrial Organization, Volume 9, Issue 1, p. 41-56, 1994.

CALLEJON, M.; SEGARRA, A. Business dynamics and efficiency in industries and regions. The case of Spain. Small Business Economics. 13(4), p.253-271, 1999.

CALLEJON, M.; ORTÚN, V. La caja negra de la dinámica empresarial. Investigaciones Regionales. Asociación Española de Ciencia Regional. n.15 p.167-189, 2009. 
CARVALHO, K.; FONSECA, L.F. O perfil da Demografia de Empresas no Brasil. Texto para discussão UFF/Economia. Universidade Federal Fluminense. TD 248, 2008.

CARVALHO, M.; ANDREOZZI, V.; CODEÇO, C.; CAMPOS, D.; BARBOSA, M.T.; SHIMAKURA, S. Análise de sobrevivência. Teoria e aplicações em saúde. Editora Fiocruz. Rio de Janeiro, 2011.

CONCEIÇÃO, O.C.; SARAIVA, M.V.; FOCHEZATTO, A.; FRANÇA, M.T.A. Brazil's Simplified Tax Regime and the longevity of Brazilian manufacturing companies: A survival analysis based on RAIS microdata, Economia, V.19(2), p.164-186, 2018.

FOTOPOULOS, G.; LOURI, H. Location and Survival of New Entry. Small Business Economics. N.4, p.311-321, 2000.

FUENTELSAZ, L.; GÓMEZ, J.; POLO, Y. Aplicaciones del análisis de supervivencia a la investigación en economía de la empresa. Cuadernos de Economia y Dirección de la Empresa. N.19, pp.81-114. 2004.

GUERRA, O.; TEIXEIRA, F. A sobrevivência das pequenas empresas no desenvolvimento capitalista. Revista de Economia Política, vol.30, n. 1(117), pp. 124-139, janeiromarço/2010.

INSTITUTO BRASILEIRO DE GEOGRAFIA E ESTATISTICA - IBGE. Demografia das empresas. Estudos e pesquisas. Informação econômica. N. 25, 2013.

KAPLAN, E. L.; MEIER, P. Nonparametric Estimation from Incomplete Observations. Journal of the American Statistical Association, V. 53, N. 282, p. 457-481, 1958.

KLEINBAUM, D.; KLEIN, M. Survival Analysis. A Self-Learning Text. Springer, Terceira edição, 2012.

LOPEZ-GARCIA, P; PUENTE, S. Business Demography in Spain: Determinants of firm survival. Banco de España. Documentos de Trabajo, № 0608. 2006.

MARTINEZ, A. F. Determinantes de la supervivencia de empresas industriales en el área metropolitana de Cali 1994-2003. Centro Regional de Estudios Económicos. Cali. n.41, pp.1-39, 2006.

MOLINERO, LUIS. Modelos de regresión de Cox para el tiempo de supervivencia. Sociedad española de hipertensión. Liga española para la lucha contra la hipertensión arterial, 2001.

RESENDE, M., CARDOSO, V., FAÇANHA, L.O. Determinants of survival of newly created SMEs in the Brazilian manufacturing industry: an econometric study. Empirical Economics. v. 50 p.1255-1274. 2016.

SARMENTO, E.; NUNES, A. Análise comparativa de sobrevivência empresarial: o caso da região norte de Portugal. Estudos Regionais. Revista portuguesa de estudos regionais. $n$. 25/26, 2011.

SEGARRA, A.; CALLEJON, M. New firms survival and market turbulence: new evidence from Spain. Review of Industrial Organization, No. 20(1), p.1-14, 2002.

SERVIÇO BRASILEIRO DE APOIO ÀS MICRO E PEQUENAS EMPRESAS -SEBRAE. Sobrevivência das empresas no Brasil. Outubro, 2016. 
SUAZO, G.; PÉREZ, J. Demografia de Empresas em Chile. Estudios económicos estadísticos. Banco Central de Chile, №108, 2014.

TSVETKOVA, A.; THILL, J.C. Metropolitan innovation, firm size, and business survival in high-tech industry. Small Business Economics. N.43 p. 661-676, 2014.

VALOR ECONOMICO. IBGE: Metade das empresas fecha as portas no Brasil após quartos anos. 04 de setembro de 2015.

WENNBERG, K.; LINDQVIST, G. The effect of cluster on the survival and performance of new firms. Small Business Economics. N.34. p.221-241, 2010.

\section{NOTAS DE AUTOR}

\section{CONTRIBUIÇÃO DE AUTORIA}

Carlos Cespedes - Concepção, Coleta de dados, Análise de dados, Elaboração do manuscrito, revisão e aprovação da versão final do trabalho.

Adelar Fochezatto - Concepção, Elaboração do manuscrito, Coleta de dados, Participação ativa da discussão dos resultados, Revisão e aprovação da versão final do trabalho.

Leandro Justino Pereira Veloso - Coleta de dados.

\section{FINANCIAMENTO}

Conselho Nacional de Desenvolvimento Cientifico e Tecnológico (CNPq)

Coordenação de Aperfeiçoamento de Pessoal de Nível Superior (CAPES)

\section{CONSENTIMENTO DE USO DE IMAGEM}

Não se aplica

\section{APROVAÇÃO DE COMITÊ DE ÉTICA EM PESQUISA}

Não se aplica

\section{CONFLITO DE INTERESSES}

Não se aplica

\section{LICENÇA DE USO}

Este artigo está licenciado sob a Licença Creative Commons CC-BY. Com essa licença você pode compartilhar, adaptar, criar para qualquer fim, desde que atribua a autoria da obra.

\section{HISTÓRICO}

Recebido em: 03-03-2018

Aprovado em: 09-06-2020 